\title{
A CLASS OF ABELIAN GROUPS
}

\author{
II. T. TUTTE
}

1. Introduction. If $M$ is any finite set we define a chain on $M$ as a mapping $f$ of $M$ into the set of ordinary integers. If $a \in M$ then $f(a)$ is the coefficient of $a$ in the chain $f$. The set of all $a \in M$ such that $f(a) \neq 0$ is the domain $|f|$ of $f$. If $|f|$ is null, that is if $f(a)=0$ for all $a$, then $f$ is the zero chain on $M$. If $M$ is null it is convenient to say that there is just one chain, a zero chain, on $M$.

The sum $f+g$ of two chains $f$ and $g$ on $M$ is a chain on $M$ defined by the following rule:

$$
(f+g)(a)=f(a)+g(a),
$$

$a \in M$.

If $M$ is null we take this to mean that the sum of the zero chain on $M$ with itself is again the zero chain on $M$.

With this definition of addition the chains on $M$ are the elements of an additive Abelian group $A(M)$. The zero element of $A(M)$ is the zero chain on $M$ and the negative in $A(M)$ of a chain $f$ on $M$ is obtained from $f$ by multiplying each coefficient $f(a)$ by -1 . We define a chain-group on $M$ as any subgroup of $A(M)$.

Let $N$ be any chain-group on $M$. A chain $f$ of $N$ is an elementary chain of $N$ (written $f$ elc $N$ ) if it is non-zero and there is no non-zero $g \in N$ such that $|g|$ is a proper subset of $|f|$. If in addition the coefficients of $f$ are restricted to the values 0,1 and -1 we say that $f$ is a primitive chain of $N$. We note that the negative of a primitive chain of $N$ is another primitive chain of $N$.

We call $N$ regular if for each elementary chain $f$ of $N$ there exists a primitive chain $g$ of $N$ such that $|g|=|f|$.

In this paper we study the properties of regular chain-groups. We find in particular that any finite graph has two associated regular chain-groups, and we relate the structure of these chain-groups to that of the graph. In discussing graphs we use the definitions and notation laid down in the introduction to (4).

2. Gycles and coboundaries on a graph. Let $G$ be any finite graph.

If $S \subseteq E(G)$ we denote by $G . S$ that subgraph of $G$ whose edges are the members of $S$ and whose vertices are the ends in $G$ of the members of $S$. We denote by $G: S$ that subgraph of $G$ whose edges are the members of $S$ and whose vertices are all the vertices of $G$. Clearly $G . S$ may be derived from $G: S$ by suppressing its isolated vertices, that is the vertices not ends of edges of $G: S$.

We denote by $G \operatorname{ctr} S$ the graph whose vertices are the components of $G$ : $(E(G)-S)$ and whose edges are the members of $S$, the ends in $G \operatorname{ctr} S$ of an

\footnotetext{
Received October 26, 1954; in revised form May 13, 1955.
} 
edge $A$ being those components of $G:(E(G)-S)$ which contain as vertices the ends of $A$ in $G$. We may regard $G \operatorname{ctr} S$ as obtained from $G$ by contracting each component of $G:(E(G)-S)$ to a single point. We denote by $G \times S$ the graph obtained from $G$ ctr $S$ by suppressing its isolated vertices. These vertices are clearly those components of $G$ whose edges all belong to $E(G)-S$.

If $S$ is the set of edges of a circular path $P$ in $G$ we denote the graph $G . S$ by $G(P)$ and call it a circuit of $G$.

We call a graph a bond if it has just two vertices, no loops, and at least one link. Each link of course has the two vertices as its ends. A bond of $G$ is a graph of the form $G \times S$ which is a bond.

Now let an orientation of $G$ be given and let it be described by a function $\eta(A, a)$ as in (4). We refer to chains on $V(G)$ and $E(G)$ as 0 -chains and 1-chains on $G$ respectively. We define their boundaries and coboundaries in the usual way. Thus the boundary $\partial f$ of a 1 -chain $f$ is given by

$$
(\partial f)(a)=\sum_{A \in E(G)} \eta(A, a) f(A),
$$

and the coboundary $\delta g$ of a 0 -chain $g$ by

$$
(\delta g)(A)=\sum_{a \in V(G)} \eta(A, a) g(a) .
$$

If $E(G)$ is null we take $(2.1)$ to mean that $\partial f$ is the zero chain on $V(G)$. Similarly if $V(G)$ is null $\delta g$ is the zero chain on $E(G)$. A cycle on $G$ is a 1-chain whose boundary is the zero chain on $V(G)$.

The set of all cycles on the oriented graph $G$ is clearly a chain-group $\Gamma(G)$ on $E(G)$. Another chain-group on $E(G)$ is the set $\Delta(G)$ of the coboundaries of the 0 -chains on $G$. We proceed to show that $\Gamma(G)$ and $\Delta(G)$ are regular.

(2.3) Let $G . S$ be any circuit of $G$. Then there is a primitive chain $g$ of $\Gamma(G)$ such that $|g|=S$.

Proof. There is a circular path $P=\left(a_{0}, A_{1}, \ldots, A_{r}, a_{0}\right)$ in $G$ such that $G(P)=G$.S. Let $g$ be a 1 -chain of $G$ defined as follows:

(i) If $A \notin S$ then $g(A)=0$,

(ii) $g\left(A_{i}\right)=1$ or -1 according as $a_{i-1}$ is or is not the positive end of $A_{i}(0<i \leqslant r)$.

Applying (2.1) we find that $\partial g$ is a zero chain. Hence $g \in \Gamma(G)$.

If $g$ is not an elementary chain of $\Gamma(G)$ there exists $k \in \Gamma(G)$ such that $|k|$ is a non-null proper subset of $S$. Then $S$ has at least two elements. Hence by the definition of a circuit the elements of $|k|$ are links of $G$ and some vertex of $G .|k|$ is an end of only one of them. This vertex must have a non-zero coefficient in $\partial k$, which is impossible. Accordingly $g$ is elementary, and therefore primitive since its coefficients are restricted to the values 0,1 , and -1 .

(2.4) Suppose $S \subseteq E(G)$. Then $S$ is the domain of an elementary chain of $\Gamma(G)$ if and only if $G . S$ is a circuit of $G$. 
Proof. Suppose $g$ elc $\Gamma(G)$. We show that there is a circuit $G . S$ of $G$ such that $S \subseteq|g|$. If $G .|g|$ has a loop this result is trivial. If not, each vertex of $G .|g|$ is an end of at least two links of $G .|g|$, by (2.1). Hence, starting at an arbitrary vertex $a_{0}$ of $G \cdot|g|$, we can construct a path

$$
P=\left(a_{0}, A_{1}, a_{1}, A_{2}, a_{2}, \ldots\right)
$$

of arbitrary length in $G .|g|$ such that $A_{i}$ and $A_{i+1}$ are distinct for each $i$ such that both exist as terms of $P$. We continue the path until some vertex $b$ is repeated. Then the part of $P$ extending from the first to the second occurrence of $b$ is a circular path in $G .|g|$ defining a circuit $G$. S such that $S \subseteq|g|$.

By (2.3) there exists $k \in \Gamma(G)$ such that $|k|=S \subseteq|g|$. Since $g$ elc $\Gamma(G)$ it follows that $|g|=S$. Thus $G$. $|g|$ is a circuit of $G$.

Since the converse result is contained in (2.3) the Theorem follows.

(2.5) $\Gamma(G)$ is a regular chain-group.

Proof. Suppose $f$ elc $\Gamma(G)$. By (2.4) there is a circuit $G . S$ of $G$ such that $|f|=S$. Hence by (2.3) there is a primitive chain $g$ of $\Gamma(G)$ such that $|g|=S=|f|$.

(2.6) Let $G \times S$ be any bond of $G$. Then there is a primitive chain $g$ of $\Delta(G)$ such that $|g|=S$.

Proof. There are two distinct components $X$ and $Y$ of $G:(E(G)-S)$ such that in $G$ each edge of $S$ has one end in $X$ and one in $Y$. Let $f$ be the 0 -chain on $G$ such that $f(a)=1$ if $a$ is a vertex of $X$ and $f(a)=0$ otherwise. Write $g=\delta f$. Then $|g|=S$ by (2.2). Further the coefficients of $G$ are restricted to the values 0,1 , and -1 .

If $g$ is not an elementary chain of $\Delta(G)$ there exists $k \in \Delta(G)$ such that $|k|$ is a non-null proper subset of $S$. Then $X$ and $Y$ are subgraphs of the same component, $Z$ say, of $G:(E(G)-|k|)$. There is a 0 -chain $f$ on $G$ such that $k=\delta f$. Since each edge of $|k|$ has both its ends in $Z$ there are two vertices of $Z$ having different coefficients in $f$. Since $Z$ is connected it must have a link $B$ whose ends have different coefficients in $f$. But then

$$
k(B)=(\delta f)(B) \neq 0
$$

by (2.2), which is impossible. Accordingly $g$ is elementary, and therefore primitive since its coefficients are restricted to the values 0,1 , and -1 .

(2.7) Suppose $S \subseteq E(G)$. Then $S$ is the domain of an elementary chain of $\Delta(G)$ if and only if $G \times S$ is a bond of $G$.

Proof. Suppose $g$ elc $\Delta(G)$. There is a 0 -chain $f$ on $G$ such that $g=\delta f$. Since $g$ is non-zero there is, by (2.2), a link $A$ of $G$ with ends $a$ and $b$ such that $f(a) \neq f(b)$. Write $f(a)=x$. Let $W$ be the set of all $c \in V(G)$ such that $f(c)=x$. Let $G[U]$ be that component of $G[W]$ which has $a$ as a vertex. (Here we use the notation of (4)). Let $S$ be the set of all links of $G$ having just one end in $G[U]$. Then $A \in S$. Moreover $S \subseteq|g|$, by $(2.2)$. 
Now $G[U]$ is one component of $G:(E(G)-S)$. Let $Z$ be the component of $G:(E(G)-S)$ which has $b$ as a vertex and let $T$ be the set of all links of $G$ having just one end in $Z$. Then $A \in T \subseteq S$. Let $f^{\prime}$ be that 0 -chain on $G$ in which the vertices of $Z$ have coefficient 1 and all other vertices of $G$ have coefficient 0 . Then

$$
A \in\left|\delta f^{\prime}\right|=T \subseteq S \subseteq|g|,
$$

by (2.2). Hence $\left|\delta f^{\prime}\right|=|g|$, since $g$ elc $\Delta(G)$, and therefore $T=S=|g|$.

We now see that each edge of $S$ has one end in $G[U]$ and one in $Z$. Hence $G \times S$, that is $G \times|g|$, is a bond of $G$.

Since the converse result is contained in (2.6) the theorem follows.

(2.8) $\Delta(G)$ is a regular chain-group.

Proof. Suppose $f$ elc $\Delta(G)$. By (2.7) there is a bond $G \times S$ of $G$ such that $|f|=S$. Hence by $(2.6)$ there is a primitive chain $g$ of $\Delta(G)$ such that $|g|=S=$ $|f|$.

3. Some operations on chain-groups. Let $N$ be any chain-group on a set $M$. Let a subset $S$ of $M$ be chosen and let the coefficient of each member of $S$ in each chain of $N$ be multiplied by -1 . The resulting chains are clearly the elements of a chain-group $N^{\prime}$ on $M$. We say that $N^{\prime}$ is obtained from $N$ by reorienting the members of $S$.

Suppose $M$ is the set of edges of an oriented graph $G$. By reorienting the members of $S$ in $G$ we mean interchanging positive and negative ends for each edge of $G$ in $S$. By (2.1) and (2.2) the effect of this operation on the chaingroups $\Gamma(G)$ and $\Delta(G)$ is to reorient the members of $S$ in each of them.

Properties of chain-groups which are invariant under reorientation are of special interest. Clearly one such property is that of regularity. We note also that the class of domains of elementary chain-groups is invariant under reorientation. In the case of $\Gamma(G)$ and $\Delta(G)$ the invariant properties correspond to properties of the underlying unoriented graph.

If $f \in N$ we define the restriction $f . S$ of $f$ to $S$ as that chain on $S$ in which each $a \in S$ has the same coefficient as in $f$.

The restrictions to $S$ of the chains of $N$ are clearly the elements of a chaingroup on $S$. We denote this chain-group by $N$. S. Another chain-group on $S$ is the set of restrictions to $S$ of those chains $f$ of $N$ for which $|f| \subseteq S$. We denote this by $N \times S$. If $T \subseteq S \subseteq M$ the following identities hold:

$$
\begin{aligned}
& (N \cdot S) \cdot T=N \cdot T, \\
& (N \times S) \times T=N \times T, \\
& (N \cdot S) \times T=(N \times(M-(S-T))) \cdot T, \\
& (N \times S) \cdot T=(N \cdot(M-(S-T))) \times T .
\end{aligned}
$$

Formulae (3.1) and (3.2) follow at once from the definitions. To prove (3.3) we observe that each side is the set of restrictions to $T$ of those chains $f$ of $N$ 
for which $|f| \cap(S-T)$ is null. We obtain (3.4) by writing $M-(S-T)$ for $S$ in (3.3).

\section{(3.5) If $N$ is regular then $N$.S and $N \times S$ are regular.}

Proof. It is clear that the elementary and primitive chains of $N \times S$ are the restrictions to $S$ of those elementary and primitive chains respectively of $N$ whose domains are subsets of $S$. Hence $N \times S$ is regular.

Now suppose $f$ elc $(N . S)$. There exists $g \in N$ such that $f=g$. S. Choose such a $g$ so that $|g|$ has the least possible number of elements. Since $N$ is regular it has a primitive chain $h$ such that $|h| \subseteq|g|$. If $|h|$ does not meet $S$ we can by adding $h$ or $-h$ to $g$ a sufficient number of times obtain $g^{\prime} \in N$ such that $g^{\prime} . S=f$ and $\left|g^{\prime}\right|$ is a proper subset of $|g|$, contrary to the definition of $g$. We deduce that there is a non-zero chain $k=h . S$ of $N . S$ whose coefficients are restricted to the values 0,1 , and -1 and which satisfies $|k| \subseteq|h|$. Since $f$ elc $(N . S)$ the chain $k$ satisfies $|k|=|f|$ and is primitive. Thus $N . S$ satisfies the definition of a regular chain-group.

4. Dendroids and representative matrices. If $f$ is a chain on a finite set $M$ and $n$ is an integer we denote by $n f$ the chain obtained from $f$ by multiplying each coefficient by $n$. It is clear that any chain-group containing $f$ as an element contains also $n f$.

Let $N$ be any chain-group on a finite set $M$.

We define a dendroid of $N$ as a subset $D$ of $M$ such that $D$, but no proper subset of $D$, meets the domain of every non-zero chain of $N$. If the only element of $N$ is the zero chain then the null subset of $M$ is the only dendroid of $N$. In every other case $M$ meets the domain of every non-zero chain of $N$ and therefore some subset of $M$ is a dendroid of $N$.

Suppose that $D$ is a dendroid of $N$ and that $a \in D$. There exists $f \in N$ such that $|f|$ is non-null and $|f| \cap(D-\{a\})$ is null. It follows that $|f| \cap D=\{a\}$ and hence that $f(a) \neq 0$. We can clearly choose $f$ so that $f(a)$ is positive. We denote a choice of $f$ for which $f(a)$ has the least possible positive value by $J^{D}$. There is only one such chain $J_{a}^{D}$, for the difference of two distinct ones would be a non-zero chain of $N$ with a domain not meeting $D$.

(4.1) $J^{D}{ }_{a}$ is an elementary chain of $N$.

Proof. Suppose $k$ is a non-zero chain of $N$ such that $|k|$ is a proper subset of $\left|J^{D}\right|$. Write $J^{D}(a)=m$ and $k(a)=n$. Since $D \cap|k|$ is non-null we have $n \neq 0$. The chain $n J^{D}{ }_{a}-m k$ of $N$ is zero since its domain does not meet $D$. Hence $|k|=\left|J_{a}^{D}\right|$, contrary to the definition of $k$.

(4.2) If $N$ is regular $J_{a}^{D}$ is primitive

Proof. By (4.1) and the regularity of $N$ there is a primitive chain $g$ of $N$ such that $|g|=\left|J^{D}{ }_{a}\right|$. Replacing $g$ by its negative if necessary we can arrange that $g(a)=1$. Then by the definition and uniqueness of $J_{a}^{D}$ we have $g=J^{D}$. 
(4.3) Suppose $N$ is regular and has a non-null dendroid D. Then for each chain $J$ of $N$ we have

$$
J=\sum_{a \in D} J(a) J^{D} a
$$

Proof. Write

$$
J^{\prime}=J-\sum_{a \in D} J(a) J_{a}^{D} .
$$

It is clear, by (4.2), that $\left|J^{\prime}\right|$ does not meet $D$. Hence $J^{\prime}$ is a zero chain.

In the rest of this section we suppose that the set $M$ is non-null. We enumerate its elements as $a_{1}, \ldots, a_{n}$. If $f$ is any chain on $M$ we refer to the row-vector $\left\{f\left(a_{1}\right), \ldots, f\left(a_{n}\right)\right\}$ as the representative vector of $f$ with respect to the chosen enumeration. Suppose $R$ is a matrix of $r$ rows and $n$ columns whose elements are integers and whose rows are linearly independent. Then the set of chains on $M$ whose representative vectors are the linear combinations of the rows of $R$ with integral coefficients are the elements of a chain-group on $M$. If this chain-group is $N$ we say that $R$ is a representative matrix of $N$ with respect to the chosen enumeration of the elements of $M$.

By the general theory of Abelian groups every chain-group on $M$ having at least one non-zero element has a representative matrix. If $N$ is a regular chaingroup of this kind we may form a representative matrix $R$ as follows. We select a dendroid $D$, necessarily non-null, and take as the rows of $R$ the representative vectors of the corresponding chains $J^{D}{ }_{a}$. It is easily seen that these vectors are linearly independent. It then follows from (4.3) that $R$ is a representative matrix of $N$. We say that the representative matrix $R$ thus constructed is associated with the dendroid $D$.

Suppose we have a representative matrix $R$ of $N$, where $N$ is not necessarily regular. Then if $S \subseteq M$ we denote by $R(S)$ the submatrix of $R$ constituted by those columns of $R$ which correspond to members of $S$. If $R(S)$ is square we denote its determinant by $\operatorname{det} R(S)$.

(4.4) Let $R$ be an r-rowed representative matrix of $N$. Then a subset $S$ of $M$ is a dendroid of $N$ if and only if it has just $r$ elements and is such that $\operatorname{det} R(S) \neq 0$.

Proof. If the rank of $R(S)$ is less than $r$ some linear combination of the rows of $R$ with integral coefficients not all zero has only zeros in the columns corresponding to members of $S$. The corresponding chain of $N$ is non-zero and has a domain not meeting $S$. Hence $S$ is not a dendroid of $N$. In particular no dendroid of $N$ has fewer than $r$ elements.

If the rank $R(S)$ is $r$ there is a subset $T$ of $S$ of just $r$ elements such that $\operatorname{det} R(T) \neq 0$. Then the rows of $R(T)$ are linearly independent. Consequently $T$ meets the domain of each non-zero chain of $N$ and so some subset of $T$ is a dendroid of $N$. This subset must be $T$ itself since a dendroid of $N$ has at least $r$ elements. We conclude that $S$ is a dendroid of $N$ if it has $r$ elements but not if it has more than $r$. 
It follows from (4.4) that all the dendroids of $N$ have the same number $r$ of elements, and that each representative matrix of $N$ has $r$ rows. We call $r$ the rank of $N$ and denote it also by $r(N)$. The Theorem does not of course apply to the case in which $N$ consists solely of the zero chain. In that case we write $r(N)=0$. Then the only dendroid of $N$ has $r(N)$ elements.

(4.5) Let $R$ be a matrix of $r$ rows and $n$ columns whose elements are integers and whose rows are linearly independent. Let $M$ be a finite set of $n$ elements. Then $R$ is a representative matrix of a regular chain-group on $M$ if and only if the determinants of its square submatrices of order $r$ are restricted to the values 0,1 , and -1 .

Proof. Suppose first that $R$ is a representative matrix of a regular chaingroup $N$ on $M$. Let $R(S)$ be any square submatrix of $R$ of order $r$.

If $S$ is not a dendroid of $N$ then $\operatorname{det} R(S)=0$, by (4.4). If $S$ is a dendroid of $N$ let $R^{\prime}$ be a representative matrix of $N$ associated with $S$, and corresponding to the same enumeration of $M$ as $R$. The rows of $R^{\prime}$ must be linear combinations of the rows of $R$ with integral coefficients. Hence there is a square matrix $P$ of order $r$ whose elements are integers and which satisfies $R^{\prime}=P R$. This implies $R^{\prime}(S)=P \times R(S)$ and hence

$$
\operatorname{det} R^{\prime}(S)=\operatorname{det} P . \operatorname{det} R(S) .
$$

Now det $R^{\prime}(S)= \pm 1$, by the definition of $R^{\prime}$. Since $P$ and $R(S)$ are matrices of integers it follows that $\operatorname{det} R(S)= \pm 1$.

Conversely, suppose that the square submatrices of $R$ of order $r$ have determinants restricted to the values 0,1 , and -1 . We fix an enumeration of the elements of $M$. There is a chain-group $N$ on $M$ whose representative matrix with respect to this enumeration is $R$.

Let $f$ be any elementary chain of $N$. Let $a$ be any member of $|f|$ and $E$ any dendroid of $N .(M-|f|)$. Then if a chain $h$ of $N$ has a domain not meeting $E \cup\{a\}$ its domain must be a subset of $|f|-\{a\}$. Since $f$ is elementary this is possible only if $h$ is zero. We conclude that some subset $D$ of $E \cup\{a\}$ is a dendroid of $N$. Since $D$ must meet $|f|$ we have $D \cap|f|=\{a\}$.

By (4.4) and the restriction imposed on $R$ we have $\operatorname{det} R(D)= \pm 1$. Hence the reciprocal of $R(D)$ is a matrix of integers. We write $R^{\prime}=(R(D))^{-1} R$. The rows of $R^{\prime}$ are linear combinations, with integral coefficients, of the rows of $R$ and are therefore representative vectors of chains of $N$. But $R^{\prime}(D)$ is a unit matrix. Hence there is a chain $g$ of $N$ such that $g(a)=1$ and $|g| \cap D=\{a\}$. Then $f-f(a) g$ is a zero chain since its domain does not meet $D$. Accordingly $f=f(a) g$.

Keeping $|f|$ fixed we may select $f$ so that the highest common factor of its non-zero coefficients is as small as possible. With this choice of $f$ the result just obtained requires $f(a)= \pm 1$. Since this is true for each $a \in|f|$ the chain $f$ is then primitive. Thus $N$ satisfies the definition of a regular chain-group. 
(4.6) Let $R$ be a matrix of $r$ rows and $n>r$ columns, whose elements are integers and in which the square submatrix $A$ constituted by some $r$ columns is unit matrix. Let the submatrix of $R$ constituted by the remaining $n-r$ columns be $B$. Let $M$ be any set of $n$ elements. Then $R$ is a representative matrix of a regular chain-group on $M$ if and only if the determinants of the square submatrices of $B$ are restricted to the values 0,1 , and -1 .

Proof. There is a 1-1 correspondence, $q$ say, between the square submatrices of $B$ and those square submatrices other than $A$ of $R$ which are of order $r$. If $C$ is a square submatrix of $B$ the corresponding submatrix $q C$ of $R$ is made up of those columns of $B$ which contain elements of $C$ and those columns of $A$ which have only zeros in the rows of $R$ meeting $C$. It is clear from this definition that $\operatorname{det} q C= \pm \operatorname{det} C$. Since the rows of $A$, and therefore the rows of $R$, are linearly independent the Theorem now follows from (4.5).

If $R$ is a representative matrix of a regular chain-group $N$ and $R^{\prime}$ is the transpose of $R$ then the number $C(N)$ of dendroids of $N$ is given by the formula

$$
C(N)=\operatorname{det}\left(R R^{\prime}\right) .
$$

This follows from (4.4) and (4.5), with the help of the well-known formula for the determinant of the product of two matrices of types $(r, n)$ and $(n, r)$.

5. Dual regular chain-groups. Two chains $f$ and $g$ on a finite set $M$ are orthogonal if

$$
\sum_{a \in M} f(a) g(a)=0 .
$$

If $M$ is null we take this to mean that the zero chain on $M$ is self-orthogonal.

If $N$ is a chain-group on $M$ then these chains on $M$ which are orthogonal to all the chains of $N$ evidently constitute a chain-group on $N$. We denote this chain-group by $N^{*}$ and call it the dual of $N$.

The zero chain-group on $M$ includes only the zero chain. The complete chain-group on $M$ includes all the chains on $M$. It is clear that these chaingroups are regular and that each is the dual of the other.

If $N$ is a regular chain-group on $M$ which is neither zero nor complete we may construct $N^{*}$ as follows. We choose arbitrarily a dendroid $D$ of $N$ and denote by $R$ a representative matrix of $N$ associated with $D$. If $r(N)=r$ we may adjust the notation so that $R(D)$ is a unit matrix occupying the first $r$ columns of $R$. We denote by $B$ the matrix constituted by the remaining columns of $R$, which we suppose $s$ in number. Now let $T$ be the matrix of $s$ rows and $r+s$ columns such that the submatrix formed by the first $r$ columns is the negative of the transpose of $B$ and the remaining $s$ columns constitute a unit matrix. Let $N_{1}$ be the chain-group on $M$ which has $T$ as a representative matrix with respect to the chosen enumeration of $M$. By (4.6) $N_{1}$ is regular. 
If $b \in M-D$ we denote by $K_{b}$ that chain of $N_{1}$ which has a row of $T$ as a representative vector and which satisfies $K_{b}(b)=1$. It is clear that $K_{b}$ is orthogonal to $J^{D}{ }_{a}$ for each $a \in D$ and each $b \in M-D$. Hence by (4.3) the chains $K_{b}$ are orthogonal to all the chains of $N$ and therefore belong to $N^{*}$. It follows that $N_{1} \subseteq N^{*}$. Now suppose $N^{*}$ has a chain $J$ not belonging to $N_{1}$. Write

$$
J^{\prime}=J-\sum_{b \in(M-D)} J(b) K_{b}
$$

The chain $J^{\prime}$ of $N$ is orthogonal to each of the chains $J^{D}{ }_{a}$ and its domain is a subset of $D$. It is therefore a zero chain. It follows that $J$ belongs to $N_{1}$, contrary to supposition. We have thus proved that $N_{1}=N^{*}$.

A similar argument in which the roles of the $J^{D}{ }_{a}$ and the $K_{b}$ are interchanged shows that $R$ is a representative matrix of $\left(N^{*}\right)^{*}$ and hence that $\left(N^{*}\right)^{*}=N$. We now have

(5.1) If $N$ is a regular chain-group then $N^{*}$ is regular and $\left(N^{*}\right)^{*}=N$.

(5.2) If $N$ is a regular chain-group on a set $M$ then the dendroids of $N^{*}$ are the complements in $M$ of the dendroids of $N$.

Proof. Let $D$ be any dendroid of $N$. If $N$ is zero or complete it is clear that $M-D$ is a dendroid of $N^{*}$. Otherwise we form the matrix $T$ as in the above construction. Since $T$ is a representative matrix of $N^{*}$ and $\operatorname{det} T(M-D)=1$ it follows from (4.4) that $M-D$ is a dendroid of $N^{*}$. Replacing $N$ by $N^{*}$ in this result, and using (5.1), we find also that if $M-D$ is a dendroid of $N^{*}$ then $D$ is a dendroid of $N$.

Suppose $N$ is a regular chain-group on a set $M$ and that $S$ is a subset of $M$. Then a chain $g$ on $S$ is orthogonal to every chain of $N . S$ if and only if it is of the form $f . S$, where $f \in N^{*}$ and $|f| \subseteq S$. We thus have

$$
(N . S)^{*}=N^{*} \times \mathrm{S} \text {. }
$$

By writing $N^{*}$ for $N$ in (5.3) and using (5.1) we obtain also

$$
(N \times S)^{*}=N^{*} . S \text {. }
$$

(5.5) Let $G$ be a finite graph and let $\Gamma(G)$ and $\Delta(G)$ be defined in terms of the same orientation of $G$. Then $(\Delta(G))^{*}=\Gamma(G)$.

Proof. If $G$ has no edge the result is trivial. In the remaining case a 1-chain $g$ on $G$ is orthogonal to all the chains of $\Delta(G)$ if and only if

$$
\sum_{A \in E(G)}\left\{g(A) \sum_{a \in V(G)} \eta(A, a) f(a)\right\}=0
$$

for arbitrary integers $f(a)$. This is so if and only if

$$
\sum_{A \in E(G)} \eta(A, a) g(A)=0
$$

for each $a \in V(G)$, that is, if and only if $g \in \Gamma(G)$. 
The dendroids of a chain-group depend only on the domains of the chains of the group and are therefore invariant under reorientation. Hence if $G$ is a finite graph and $\Delta(G)$ is its group of coboundaries with respect to some fixed orientation we may expect the dendroids of $\Delta(G)$ to be interpretable in terms of the structure of $G$ only.

If $H$ and $K$ are two subgraphs of $G$ we define their intersection $H \cap K$ as that subgraph of $G$ whose edges and vertices are the common edges and vertices respectively of $H$ and $K$. A forest is a graph which has no circuit. A tree is a connected forest. A spanning forest of $G$ is a subgraph of $G$ of the form $G: S$ whose intersection with each component of $G$ is a tree.

(5.6) Let $G$ be a finite graph with a given orientation and let $S$ be a subset of $E(G)$. Then $S$ is a dendroid of $\Delta(G)$ if and only if $G: S$ is a spanning forest of $G$.

Proof. Suppose $G: S$ is not a spanning forest of $G$. If $G: S$ has a circuit then $E(G)-S$ is not a dendroid of $\Gamma(G)$, by (2.4), and therefore $S$ is not a dendroid of $\Delta(G)$, by (5.2) and (5.5). If $G: S$ has no circuit its intersection with each component of $G$ is a forest. Hence there must be a component $H$ of $G$ such that $H \cap(G: S)$ is not connected. Let $K$ be any component of $H \cap(G: S)$. Let $f$ be the 0 -chain on $G$ such that $f(a)=1$ if $a$ is a vertex of $K$ and $f(a)=0$ otherwise. Then the chain $\delta f$ is non-zero and its domain does not meet $S$. Again we find that $S$ is not a dendroid of $\Delta(G)$.

Conversely suppose $S$ is not a dendroid of $\Delta(G)$. Assume that $G: S$ is a spanning forest of $G$. Let $f$ be any 0 -chain on $G$ such that $\delta f$ is non-zero. Then some component $H$ of $G$ has two vertices $a$ and $b$ such that $f(a) \neq f(b)$. Since $H \cap(G: S)$ is a tree there are two vertices $c$ and $d$ of $H \cap(G: S)$, joined by an edge of $S$, such that $f(c) \neq f(d)$. Hence $S$ meets $|\delta f|$. We deduce that some proper subset $T$ of $S$ is a dendroid of $\Delta(G)$. Choose $e \in S-T$ and write $Q=E(G)-T$. Now $Q$ is a dendroid of $\Gamma(G)$, by (5.2). The non-zero element $J^{Q}{ }_{e}$ of $\Gamma(G)$ satisfies $\left|J^{Q}{ }_{e}\right| \subseteq S$. Hence, by (2.4), $G: S$ has a circuit, contrary to our assumption. We deduce that in fact $G: S$ is not a spanning forest of $G$. The Theorem follows.

6. Conformity. Let $f$ and $g$ be chains on a finite set $M$. We say that $f$ conforms to $g$ if the following condition is satisfied: if $f(a) \neq 0$ then $g(a)$ is non-zero and has the same sign as $f(a)$. Conformity is clearly a transitive relation.

(6.1) If $N$ is a regular chain-group and $f$ is a non-zero chain of $N$ then there exists a primitive chain of $N$ conforming to $f$.

Proof. If possible choose $f$ so that the Theorem fails and $|f|$ has the least number of elements consistent with this condition. Since $N$ is regular it has a primitive chain $h$ such that $|h| \subseteq|f|$. Choose $a \in|h|$ so that $f(a)$ has the least possible absolute value. Replacing $h$ by its negative if necessary, we arrange that $h(a)=1$. Write $k=f-f(a) h$. Clearly $k$ conforms to $f$. If $k$ is a zero 
chain then either $h$ or $-h$ conforms to $f$. If $k$ is non-zero there is a primitive chain $g$ of $N$ conforming to $k$, and therefore to $f$, since $|k|$ is a proper subset of $|f|$. In each case the definition of $f$ is contradicted.

(6.2) If $N$ is a regular chain-group then each non-zero chain of $N$ can be represented as a sum of primitive chains of $N$ each conforming to it.

Proof. If $f \in N$ let $Z(f)$ be the sum of the absolute values of the coefficients of $f$. If possible choose a non-zero $f \in N$ for which the Theorem fails and $Z(f)$ has the least value consistent with this condition. By (6.1) there is a primitive chain $g$ of $N$ conforming to $f$. Clearly $f-g$ conforms to $f$ and $Z(f-g)<Z(f)$. By the latter result $f-g$ is either a zero chain or a sum of primitive chains of $N$ conforming to it. But chains conforming to $f-g$ conform also to $f$. Hence the Theorem is true for $f$ and we have a contradiction.

Let $f$ and $g$ be chains on a finite set $M$ and let $q$ be an integer $>1$. We say that $g$ is a $q$-representative of $f$ if the following conditions are satisfied:

(i) $g(a)=f(a)(\bmod q)$ for each $a \in M$,

(ii) $|g(a)|<q$ for each $a \in M$.

(6.3) If $N$ is a regular chain-group on a set $M$ and $f \in N$ then for each integer $q>1$ some q-representative of $f$ is a chain of $N$.

Proof. Let $f$ be any chain of $N$ and $q$ any integer $>1$. There is at least one $g \in N$ satisfying (i). For any such $g$ we denote by $Y(g)$ the number of elements $a$ of $M$ for which $|g(a)| \geqslant q$. We choose a particular $g$ satisfying (i) so that $Y(g)$ has the least possible value.

If $Y(g)>0$ choose $b \in M$ such that $|g(b)| \geqslant q$. By (6.2) there is a primitive chain $h$ of $N$ conforming to $g$ and such that $h(b)= \pm 1$. Write $g^{\prime}=g-q h$. Clearly $g^{\prime}$ satisfies (i). Moreover we have

(2) if

$$
\begin{gathered}
\left|g^{\prime}(b)\right|<|g(b)|, \\
|g(a)|<q \text { then }\left|g^{\prime}(a)\right|<q .
\end{gathered}
$$

If $\left|g^{\prime}(b)\right| \geqslant q$ we repeat the process with $g^{\prime}$ replacing $g$ and with the same choice of $b$. Proceeding in this way we eventually obtain a chain $g_{1}$ of $N$ which satisfies (i) and is such that $Y\left(g_{1}\right)<Y(g)$. This contradicts the definition of $g$. We conclude that $Y(g)=0$, that is, $g$ is a $q$-representative of $f$.

This Theorem is proved for the cycle-group of an oriented graph in (3). For applications of it to the theory of graphs see (3) and (4, pp. 83-84).

7. Homomorphisms. Let $N$ be a regular chain-group on a set $M$. A homomorphism of $N$ (into $I$ ) is a mapping $\phi$ of $N$ into the set $I$ of integers such that

$$
\phi(f+g)=\phi(f)+\phi(g)
$$

for arbitrary chains $f$ and $g$ of $N$. This implies that $\phi(f)=0$ if $f$ is the zero chain. Hence $\phi(-f)=-\phi(f)$ for each $f \in N$. 
For arbitrary chains $f$ and $g$ on $M$ we write

$$
(f \cdot g)=\sum_{a \in M} f(a) g(a) .
$$

If $M$ is null we take this to mean $(f . g)=0$.

A solution of $\phi$ is a chain $g$ on $M$ such that $(f . g)=\phi(f)$ for each $f \in N$. In this section we study the solutions of the homomorphisms of $N$. We need the following definitions.

If $f \in N$ we define $P(f)$ as the set of all $a \in M$ such that $f(a)>0$. We then write

$$
\beta(f)=\sum_{a \in P(f)} f(a) .
$$

If $P(f)$ is null we take $\beta(f)$ to be 0 . We call $f$ a positive chain of $N$ if $P(f)=|f|$.

(7.4) Let $\phi$ be any homomorphism of $N$ and $a$ any element of $M$. Then either $\{a\}$ is the domain of a chain of $N$ or there is a homomorphism $\phi_{a}$ of $N .(M-\{a\})$ such that

$$
\phi_{a}(f .(M-\{a\}))=\phi(f)
$$

for eachf $\in N$.

Proof. Suppose $\{a\}$ is not the domain of a chain of $N$. Then no two distinct chains of $N$ have the same restriction to $M-\{a\}$, for otherwise the domain of their difference would be $\{a\}$. Hence there is a unique mapping $\phi_{a}$ of $N$. $(M-\{a\})$ into $I$ such that

$$
\phi_{a}(f .(M-\{a\}))=\phi(f)
$$

for each $f \in N$. It is easily verified that $\phi_{a}$ is a homomorphism.

(7.5) If $\phi$ is any homomorphism of $N$ and $f$ is a chain of $N$ such that $\phi(f)>\beta(f)$ then there is a primitive chain $g$ of $N$ conforming to $f$ such that $\phi(g)>\beta(g)$.

Proof. The chain $f$ is necessarily non-zero. Hence by (6.2) it is a sum $f_{1}+f_{2}+\ldots+f_{s}$ of primitive chains $f_{i}$ of $N$ conforming to $f$. If the Theorem is false, $\phi\left(f_{i}\right) \leqslant \beta\left(f_{i}\right)$ for each of these. Then by addition we have $\phi(f) \leqslant \beta(f)$, contrary to hypothesis.

(7.6) If $\phi$ is any homomorphism of $N, a$ an element of $M$, and $f$ a chain of $N$ such that $f(a) \neq 0$ and

$$
\phi(f)-\beta(f)+\epsilon f(a)>0,
$$

where $\epsilon$ is 1 or -1 , then there is a primitive chain $g$ of $N$ conforming to $f$ such that either $\phi(g)>\beta(g)$ or $g$ satisfies the equations $\phi(g)=\beta(g)$ and $g(a)=\epsilon$.

Proof. By (6.2) $f$ is a sum $f_{1}+f_{2}+\ldots+f_{s}$ of primitive chains $f_{i}$ of $N$ each conforming to $f$. There must be just $|f(a)|$ of these such that $\left|f_{i}(a)\right|=1$. 
If one of the $f_{i}$ satisfies $\phi\left(f_{i}\right)>\beta\left(f_{i}\right)$ the Theorem is true. In the remaining case we have by addition $\phi(f)-\beta(f) \leqslant 0$. But

$$
\phi(f)-\beta(f)+\epsilon f(a)>0 .
$$

One consequence of this is that $f(a)$ has the same sign as $\epsilon$, whence it follows that the $|f(a)|$ chains $f_{i}$ satisfying $\left|f_{i}(a)\right|=1$ satisfy also $f_{i}(a)=\epsilon$. Another consequence is that at most $|f(a)|-1$ of the chains $f_{i}$ satisfy

$$
\phi\left(f_{i}\right)-\beta\left(f_{i}\right)<0 .
$$

Combining these results we see that one of the chains $f_{i}$ satisfies both $\phi\left(f_{i}\right)=$ $\beta\left(f_{i}\right)$ and $f_{i}(a)=\epsilon$.

(7.7) Let $\phi$ be any homomorphism of $N$. Then in order that $\phi$ shall have a solution whose coefficients are restricted to the values 0 and 1 it is necessary and sufficient that $\phi(g) \leqslant \beta(g)$ for each primitive chain $g$ of $N$.

Proof. Let us call a solution of a homomorphism limited if its coefficients are restricted to 0 and 1 .

The theorem is trivially true if $M$ is null. Assume as an inductive hypothesis that it is true whenever the number $\alpha(M)$ of elements of $M$ is less than some positive integer $q$. Consider the case $\alpha(M)=q$.

Suppose there is a primitive chain $g$ of $N$ such that $\phi(g)>\beta(g)$. Then any chain $h$ on $M$ with coefficients restricted to the values 0 and 1 satisfies

$$
(g \cdot h) \leqslant \beta(g)<\phi(g) .
$$

Hence no limited solution of $\phi$ exists.

Conversely suppose $\phi$ has no limited solution. Assume there is no primitive chain $g$ of $N$ such that $\phi(g)>\beta(g)$. It may happen that each $a \in M$ constitutes the domain of a chain of $N$. Then, since $N$ is regular, there is for each $a \in M$ a chain $f_{a}$ of $N$ such that $f_{a}(a)=1$ and $f_{a}(b)=0$ if $b \neq a$. We define a chain $h$ on $M$, with coefficients restricted to the values 0 and 1 , by writing $h(a)=\phi\left(f_{a}\right)$ for each $a \in M$. Then for each $f \in N$ we have

$$
\begin{aligned}
(f . h) & =\left(\left(\sum_{a \in M} f(a) f_{a}\right) \cdot h\right)=\sum_{a \in M} f(a)\left(f_{a} \cdot h\right) \\
& =\sum_{a \in M} f(a) \phi\left(f_{a}\right)=\phi(f) .
\end{aligned}
$$

Thus $h$ is a limited solution of $\phi$. But this is impossible.

We deduce that there exists $a \in M$ such that $\{a\}$ is not the domain of a chain of $N$. We define $\phi_{a}$ as in (7.4). There is no limited solution of $\phi_{a}$, for such a solution would be the restriction to $M-\{a\}$ of a limited solution $d$ of $\phi$ satisfying $d(a)=0$. Hence, by the inductive hypothesis and (3.5) there exists $f \in N$ such that

$$
\phi_{a}(f .(M-\{a\}))-\beta(f .(M-\{a\}))>0 .
$$


If $f(a) \leqslant 0$ it follows that $\phi(f)-\beta(f)>0$. By (7.5) this is contrary to our assumptions. Hence $f(a)>0$ and we have

$$
\phi(f)-\beta(f)+f(a)>0 .
$$

By (7.6) and our assumptions it follows that there is a chain $j$ of $N$ such that

$$
\phi(j)-\beta(j)=0 \text { and } j(a)=1 .
$$

Now let $\psi$ be the homomorphism of $N$ defined by $\psi(f)=\phi(f)-f(a)$ for each $f \in N$. The homomorphism $\psi_{a}$ of $N$. (M-\{a\}) has no limited solution, for such a solution would be a restriction to $M-\{a\}$ of a limited solution $d$ of $\phi$ such that $d(a)=1$. Hence by the inductive hypothesis and (3.5) there exists $f \in N$ such that

$$
\phi(f)-f(a)-\beta(f .(M-\{a\}))>0 .
$$

If $f(a) \geqslant 0$ this gives $\phi(f)-\beta(f)>0$. By (7.5) this is contrary to our assumptions. Hence $f(a)<0$ and

$$
\phi(f)-\beta(f)-f(a)>0 .
$$

By (7.6) and our assumptions it follows that there exists $k \in N$ such that

$$
\phi(k)-\beta(k)=0 \text { and } k(a)=-1 .
$$

It follows from (i) and (ii) that $\phi(j+k)-\beta(j+k)>0$. This is contrary to our assumptions, by (7.5). This completes the proof for the case $\alpha(M)=q$.

The general theorem follows by induction.

(7.8) Let $\phi$ be any homomorphism of $N$. Then $\phi$ has a solution whose coefficients are all non-negative if and only if $\phi(f) \geqslant 0$ for each positive primitive chain $f$ of $N$.

Proof. $\quad N$ has only a finite number of primitive chains. Hence we can find an integer $q>0$ such that $\phi(f)<q$ for each primitive chain $f$ of $N$.

Choose a set $U$, the union of $\alpha(M)$ disjoint sets $U_{a}$, one for each $a \in M$. Each $U_{a}$ is to have just $q$ elements. If $k \in N$ we denote by $k^{\prime}$ the chain on $U$ in which the coefficient of each element of $U_{a}$ is $k(a)$, for each $a \in M$. The chains $k^{\prime}$ constitute a chain-group $N^{\prime}$ on $U$. Elementary and primitive chains of $N^{\prime}$ correspond respectively to elementary and primitive chains of $N$. Hence $N^{\prime}$ is regular. There is a homomorphism $\phi^{\prime}$ of $N^{\prime}$ such that $\phi^{\prime}\left(k^{\prime}\right)=\phi(k)$ for each $k \in N$.

If $\phi(f)<0$ for some positive primitive chain $f$ of $N$ it is clear that $\phi$ has no solution whose coefficients are all non-negative.

In the remaining case we have $\phi^{\prime}\left(g^{\prime}\right) \leqslant \beta\left(g^{\prime}\right)$ for each primitive chain $g^{\prime}$ of $N^{\prime}$. This follows from the definition of $N^{\prime}$ if $\beta\left(g^{\prime}\right)>0$. In the remaining case $-g^{\prime}$ corresponds to a positive chain $-g$ of $N$, and so $\phi^{\prime}\left(g^{\prime}\right)=-\phi(-g) \leqslant 0=$ 
$\beta\left(g^{\prime}\right)$. Hence by $(7.7) \phi^{\prime}$ has a limited solution $h^{\prime}$. There is a corresponding solution $h$ of $\phi$ defined by

$$
h(a)=\sum_{c \in U_{a}} h^{\prime}(c), \quad a \in M .
$$

The coefficients in $h$ are all non-negative.

(7.9) If a $\in M$ then either $N$ or $N^{*}$ has a positive primitive chain $f$ such that $a \in|f|$.

Proof. By (6.2) it is sufficient to show that either $N$ or $N^{*}$ has a positive chain $f$ such that $a \in|f|$.

Let $\phi$ be the homomorphism of $N$ such that $\phi(f)=-f(a)$ for each $f \in N$. If $\{a\}$ is the domain of a chain of $N$ the Theorem is clearly true. If not we define $\phi_{a}$ as in (7.4). Then, if $\phi_{a}$ has a solution $h^{\prime}$ with coefficients all nonnegative, let $h$ be the chain on $M$ such that $h(a)=1$ and $h .(M-\{a\})=h^{\prime}$. Then $(f . h)=0$ for each $f \in N$ and so $h$ is a positive chain of $N^{*}$. If no such solution $h^{\prime}$ exists, then by (7.8) there exists $f \in N$ such that $f .(M-\{a\})$ is positive and $-f(a)=\phi(a)<0$. Then $f$ is a positive chain of $N$ such that $a \in|f|$. In either case the Theorem is true.

8. Some applications to graph theory. Let $G$ be a graph taken with a fixed orientation.

A directed bond of $G$ is a bond $G \times S$ of $G$ such that the positive ends of the edges of $S$ all belong to the same component of $G:(E(G)-S)$. A directed circuit of $G$ is a circuit $G$. S of $G$ defined by a circular path in which each edge is immediately succeeded by its positive end. Using (2.4) and (2.7) we may verify that the subsets $S$ of $E(G)$ such that $G \times S$ is a directed bond or $G . S$ a directed circuit of $G$, are the domains of the positive primitive chains of $\Delta(G)$ and $\Gamma(G)$ respectively. If we apply this to (5.5) and (7.9) we obtain the following graph-theoretical result.

(8.1) Any edge of $G$ is an edge of some directed bond or of some directed circuit of $G$.

In conclusion we show how (7.8) may be applied to obtain a known theorem concerning the 1 -factors of even graphs $(1 ; 2)$.

We suppose henceforth that $G$ is even, that is, the set $V(G)$ falls into two disjoint subsets $V_{1}$ and $V_{2}$ such that each edge of $G$ has one end in $V_{1}$ and the other in $V_{2}$. We fix an orientation by taking the positive end of each edge in $V_{2}$. If $a \in V(G)$ we write $\sigma(a)=1$ or -1 according as $a$ is in $V_{2}$ or $V_{1}$. We call $G$ balanced if each component has the same number of vertices in $V_{1}$ as in $V_{2}$. The decomposition $\left\{V_{1}, V_{2}\right\}$ of $V(G)$ is unique within each component of $G$, apart from the order of $V_{1}$ and $V_{2}$. Hence if $G$ is balanced for one such decomposition it is balanced for all of them.

A 1-factor of $G$ is a subgraph $G: F$ of $G$ such that each vertex of $G$ is an end of just one edge of $F$. It is clear that a graph which is not balanced has no 1 -factor. For balanced graphs we prove the following theorem. 
(8.2) Suppose $G$ balanced. Then $G$ has a 1 -factor if and only if there is no subset $U$ of $V_{1}$ such that the set of all vertices of $V_{2}$ joined by edges of $G$ to vertices of $U$ has fewer members than $U$.

Proof. If such a subset $U$ of $V_{1}$ exists it is clear that $G$ has no 1-factor.

Conversely suppose $G$ has no 1-factor. Then for each $g \in \Delta(G)$ we write

$$
\phi(g)=\sum_{a \in V(G)} \sigma(a) f(a)
$$

where $f$ is any 0 -chain on $G$ such that $\delta f=g$. If $f_{1}$ and $f_{2}$ are two such 0 -chains and $\phi_{1}(g)$ and $\phi_{2}(g)$ are the corresponding values of $\phi(G)$ we have

$$
\phi_{1}(g)-\phi_{2}(g)=\sum_{a \in V(G)} \sigma(a)\left(f_{1}(a)-f_{2}(a)\right) .
$$

Now $\delta\left(f_{1}-f_{2}\right)=\delta\left(f_{1}\right)-\delta\left(f_{2}\right)$, which is the zero 1-chain on $G$. Hence by $(2.2)$ $f_{1}(a)-f_{2}(a)$ is the same for all vertices $a$ of any one component of $G$. Since $G$ is balanced it follows from (ii) that $\phi_{1}(g)=\phi_{2}(g)$. Hence $\phi(g)$ is uniquely defined for each $g \in \Delta(G)$. It is now clear that $\phi$ is a homomorphism of $\Delta(G)$.

Suppose $\phi$ has a solution $h$ whose coefficients are all non-negative. By considering the coboundaries $\delta(f)$ such that $f$ has only one non-zero coefficient we find that

$$
\sum_{A \in E(G)} \eta(A, a) h(A)=\sigma(a)
$$

for each $a \in V(G)$. But $\eta(A, a) \sigma(a) \geqslant 0$ for each $a, A$. It follows that $h(A)$ is 0 or 1 for each $A$ and that the edges for which $h(A)=1$ define a 1 -factor of $G$. This contradicts our supposition. Hence by (7.8) there is a positive primitive chain $k$ of $\Delta(G)$ such that $\phi(k)<0$.

Now $G \times|k|$ is a directed bond of $G$. Let $C$ be the component of $G:(E(G)-$ $|k|)$ which includes the positive ends of the members of $|k|$. Let $f$ be the 0 -chain on $G$ such that $f(a)=1$ or 0 according as $a$ is or is not a vertex of $C$. By (i) we have

$$
\sum \sigma(a)<0
$$

where the summation is over the vertices of $C$. If $U$ is the set of all vertices of $C$ in $V_{1}$ it follows that $U$ is a subset of $V_{1}$ of the kind specified in the enunciation.

\section{REFERENCES}

1. P. Hall, On representation of subsets, J. London Math. Soc., 10 (1934), 26-30.

2. R. Rado, Factorization of even graphs, Quart. J. Math., 20 (1949), 95-104.

3. W. T. Tutte, On the imbedding of linear graphs in surfaces, Proc. London Math. Soc. (2), 51 (1949), 474-483.

4. - - A contribution to the theory of chromatic polynomials, Canadian J. Math., 6 (1953), 80-91.

University of Toronto 